
OP0235 INTERFERON-FREE ANTIVIRALS FOR HEPATITIS C VIRUS-ASSOCIATED CRYOGLOBULINEMIA VASCULITIS: A LONG-TERM FOLLOW-UP STUDY

P. Cacoub ${ }^{1}$, S.N. Si ahmed ${ }^{2}$, Y. ferfar' ${ }^{1}$, S. Pol ${ }^{3}$, D. Thabut ${ }^{1}$, C. Hezode ${ }^{4}$, L. Alric ${ }^{5}$, C. Comarmond ${ }^{1}$, G. Ragab ${ }^{6}$, L. Quatuccio ${ }^{7}$, M. Hegazy 6 , T. Poynard ${ }^{1}$, M. Resche Rigon ${ }^{8}$, D. Saadoun ${ }^{1}{ }^{1}$ Hôpita Pitié Salpétrière, Paris; ${ }^{2} \mathrm{CH}$ Orleans, Orleans; ${ }^{3}$ Hôpital Cochin, PARIS; ${ }^{4}$ Hôpital H Mondor, Creteil; ${ }^{5} \mathrm{CH}$ Purpan, Toulouse, France; ${ }^{6}$ Cairo university, Cairo, Egypt, ${ }^{7}$ University Hospital Udine, Udine, Italy, ${ }^{8}$ Hôpital Saint Louis, Paris, France

Background: In small-size and short term studies of hepatitis C virus (HCV)-cryoglobulinemia vasculitis (CryoVas), direct antiviral agents (DAAs) showed a better response rate and tolerance than interferon containing regimens.

Objectives: To evaluate the effectiveness and tolerance of all oral interferon-free DAA in a large CryoVas cohort with long-term follow-up.

Methods: This prospective international multicenter cohort study included 148 symptomatic HCV-CryoVas patients $(53.7 \%$ with cirrhosis and $49.3 \%$ antiviralnaïve). They all received DAA, i.e. sofosbuvir (SOF) plus daclatasvir $(n=53)$, SOF plus ribavirin $(n=51)$, SOF plus ledipasvir $(n=23)$, or SOF plus simeprevir $(n=18)$, for 12 or 24 weeks. The primary endpoint was the clinical response of CryoVas symptoms at week 12 after stopping DAAs.

Results: $106(72.6 \%)$ patients showed a complete response, $33(22.6 \%)$ a partial response and $7(4.8 \%)$ no response of CryoVas symptoms. Cryoglobulinemia was no longer found in $53.1 \%$. A sustained virological response was obtained in $97.2 \%$. Premature DAA withdrawal was noted in $4.1 \%$. Two factors were associated with a poor response: a severe form of CryoVas [OR 0.33, $95 \% \mathrm{Cl}: 0.12$ to $0.91 ; p=0.03$ ] and peripheral neuropathy [OR $0.31,95 \% \mathrm{Cl}: 0.11$ to $0.84 ; p=0.02$ ] After a median follow-up of 15.3 months, $4(2.8 \%)$ patients died. The final clearance rates of CryoVas manifestations were as follows: purpura (97.2\%), renal involvement $(91.5 \%)$, arthralgia $(85.7 \%)$, neuropathy $(77.1 \%)$ and cryoglobulinemia $(53.8 \%)$. Only SOF plus ledipasvir regimen showed significant superiority [OR $4.09,95 \% \mathrm{Cl}: 1.19$ to $19.00 ; \mathrm{p}=0.04$ ]

Conclusions: The different DAA combinations showed high response rates of HCV-CryoVas symptoms. The tolerance was good, and the mortality rate was very low. We identified prognosis factors of response to DAA.

Disclosure of Interest: P. Cacoub Consultant for: Janssen, BMS, Abbvie, GSK, Astra zeneka, Gilead, Merck, Roche, Servier, Vifor, S. N. Si ahmed Consultant for: BMS, Abbvie, Gilead, Roche, Janssen, Y. ferfar: None declared, S. Pol Consultant for: Sanofi, Novartis, Vertex, Boehringer, Janssen, BMS, Abbvie, GSK, Astra zeneka, Gilead, Merck, Roche, Servier, Vifor, D. Thabut: None declared, C. Hezode Consultant for: BMS, Merck, Abbvie, Gilead, Roche, Janssen, L. Alric Consultant for: BMS, Abbvie, Gilead, Janssen, Merck, C. Comarmond: None declared, G. Ragab: None declared, L. Quatuccio: None declared, M. Hegazy: None declared, T. Poynard: None declared, M. Resche Rigon: None declared, D. Saadoun Consultant for: medimmune, BMS, Abbvie, GSK, Astra zeneka, Gilead, Merck, Roche, Servier,

DOI: 10.1136/annrheumdis-2018-eular.2324

\section{OP0236 RISK OF CARDIOVASCULAR DISEASE AND VENOUS THROMBOEMBOLISM AMONG PATIENTS WITH INCIDENT ANCA-ASSOCIATED VASCULITIS: A 20 YEAR POPULATION-BASED COHORT STUDY}

A Berti $^{1,2}$, E. Matteson ${ }^{3}$, C.S. Crowson ${ }^{3}$, U. Specks ${ }^{4}$, D. Cornec ${ }^{5} .{ }^{1}$ Unit of Immunology, Rheumatology, Allergy and Rare Diseases, IRCCS San Raffaele Scientific Institute, Milan; ${ }^{2}$ Rheumatology Clinic, Santa Chiara Hospital, Trento, Italy; ${ }^{3}$ Division of Rheumatology; ${ }^{4}$ Division of Pulmonary and Critical Care Medicine, Mayo Clinic, Rochester, USA; ${ }^{5}$ Lymphocytes B et Autoimmunité, Université de Bretagne Occidentale, Brest, France

Background: Although modern treatments have greatly improved survival in ANCA-associated vasculitis (AAV), many patients suffer cardiovascular and thrombotic complications during long-term follow up.

Objectives: We aimed to assess the cardiovascular disease (CVD) and venous thromboembolism (VTE) risks among patients with newly diagnosed AAV.

Methods: A population-based incident AAV cohort of 58 patients diagnosed between 1996 and 2015 in Olmsted County (Minnesota, USA) was identified by medical record review. For each patient, 3 age- and sex-matched non-AAV comparators were randomly selected from the same population and assigned an index date corresponding to the AAV incidence date. Medical records of cases and comparators were reviewed for CVD events, which included cardiac events (coronary artery disease, heart failure and atrial fibrillation), cerebrovascular accidents (CVA), peripheral vascular disease (PVD) and VTE, which included deep vein thrombosis (DVT) and pulmonary embolism (PE).

Results: Baseline total cholesterol, high-density lipoprotein and current smoking rate were lower in AAV than comparators $(p=0.03, p=0.01$ and $p=0.04$, respectively), while other CVD risk factors and Framingham risk score were not significantly different between the 2 groups. CVD events developed in 13 patients and
17 comparators, corresponding to a $>3$ fold increased risk (hazard ratio [HR] 3.15; 95\% confidence interval [CI]:1.51-6.57) (figure 1). By subtypes, risks were increased for cardiac events (HR 2.96, 95\% Cl: 1.42 to 6.15) and CVA (HR 8.16, 95\% Cl: 2.45 to 27.15 ), but not for PVD. The HR for VTE was 3.26 (95\% Cl: 0.84 to 12.60 ), significantly increased for DVT (HR $6.25,95 \% \mathrm{Cl}: 1.16$ to 33.60$)$, but not for PE (HR 1.33, 95\% Cl: 0.23 to 7.54 ).

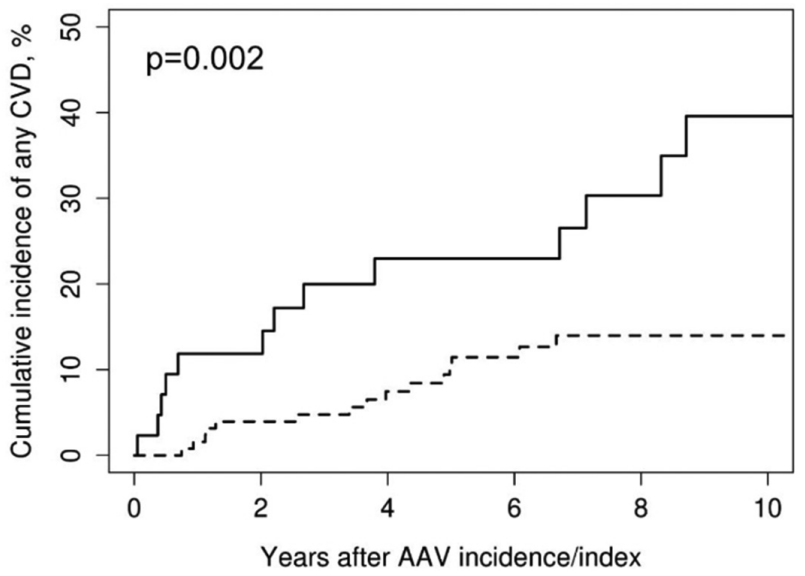

Conclusions: Despite a similar prevalence of CVD risk factors at baseline, the risk of CVD is $>3$ fold higher and for CVA 8-fold higher in patients with incident $\mathrm{AAV}$ than matched comparator subjects.

\section{REFERENCES}

[1] Avina-Zubieta JA, Mai A, et al. Risk of myocardial infarction and stroke in patients with granulomatosis with polyangiitis (Wegener's): A populationbased study. Arthritis Rheumatol 2016;68(11):2752-9.

[2] Morgan MD, et al. Increased incidence of cardiovascular events in patients with antineutrophil cytoplasmic antibody-associated vasculitides: A matched-pair cohort study. Arthritis Rheum 2009;60(11):3493-500.

Disclosure of Interest: None declared

DOI: 10.1136/annrheumdis-2018-eular.7206

\section{OP0237 A COMPARISON OF PK AND PD OUTCOMES OF TOCILIZUMAB IN GIANT CELL ARTERITIS AFTER SC AND IV DOSING}

N.L. Mallalieu ${ }^{1}$, J.H. Stone ${ }^{2}$, P. Villiger ${ }^{3}$, M. Klearman ${ }^{4}$, L. Brockwell ${ }^{5}$, S. Dimonaco ${ }^{5}$, J.E. Charion ${ }^{6} .{ }^{1}$ Roche Innovation Ctr, New York; ${ }^{2}$ Massachusetts Gen Hosp Rheumatol Unit, Harvard Med School, Boston, USA; ${ }^{3}$ Dept Rheumatology, Immunology and Allergology, U Bern, Bern, Switzerland; ${ }^{4}$ Genentech, South San Francisco, USA; ${ }^{5}$ Roche Products Ltd, Welwyn Garden City, UK; ${ }^{6}$ Roche Innovation Ctr, Basel, Switzerland

Background: Tocilizumab (TCZ), a humanised anti-interleukin-6 (IL-6) recepto monoclonal antibody, was recently approved for the treatment of patients with giant cell arteritis (GCA). Evidence was based on results of a double-blind randomised controlled trial (RCT) in GCA patients given $162 \mathrm{mg} \mathrm{TCZ}$ either weekly $(\mathrm{QW})$ or every other week (Q2W) via subcutaneous $(\mathrm{SC})$ route $\left(\mathrm{GiACTA}\right.$ trial $\left.{ }^{1}\right)$. A second RCT conducted using $8 \mathrm{mg} / \mathrm{kg}$ TCZ given intravenously (IV) every 4 weeks (Q4W) also showed positive outcomes in GCA patients. ${ }^{2}$ The double-blind dosing portion of each study lasted approximately 1 year. All three regimens (SC $162 \mathrm{mg} \mathrm{QW}$, SC $162 \mathrm{mg}$ Q2W, IV $8 \mathrm{mg} / \mathrm{kg}$ Q4W) resulted in positive outcomes for sustained remission of GCA. However, a higher benefit was noted in some key secondary efficacy outcomes with the QW vs the Q2W SC regimen. ${ }^{1}$

Objectives: To characterise the pharmacokinetics (PK) of TCZ in the GCA population and to assess the impact of the exposure differential from the three regimens on pharmacodynamic (PD) markers.

Methods: TCZ levels and PD biomarkers (soluble IL- 6 receptor [sIL-6R], IL-6, erythrocyte sedimentation rate [ESR], and C-reactive protein [CRP]) were measured using validated assays at regular intervals throughout the dosing period from all patients in each trial. A comparison of PK and PD outcomes was conducted to understand the dose exposure-response relationships.

Results: At week 52, mean trough steady state exposure $\left(\mathrm{C}_{\text {trough }}\right)$, a primary PK driver of TCZ efficacy, was highest from SC $162 \mathrm{mg} \mathrm{QW}$, followed by IV $8 \mathrm{mg} / \mathrm{kg}$ Q4W, and finally SC Q2W (figure 1). Of the PD end points, at week 52, sIL-6R levels were similar for the SC QW and IV regimens but lower for the SC Q2W regimen 